\title{
Defect-Assisted Photoinduced Halide Segregation in Mixed-Halide Perovskite Thin Films
}

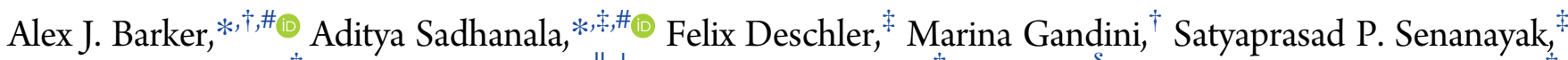
Phoebe M. Pearce, ${ }^{\ddagger}$ Edoardo Mosconi, ${ }^{\|, \perp}$ Andrew J. Pearson, ${ }^{\ddagger}$ Yue Wu, Ajay Ram Srimath Kandada, Tomas Leijtens, ${ }^{\dagger}$ Filippo De Angelis, ${ }^{\prime \prime} \perp$ @ Siân E. Dutton, ${ }^{\dagger}$ Annamaria Petrozza, ${ }^{*}{ }^{\dagger}$ (i) and Richard H. Friend ${ }^{*}$

${ }^{\dagger}$ Center for Nano Science and Technology@PoliMi, Istituto Italiano di Tecnologia, via Giovanni Pascoli 70/3, 20133 Milan, Italy ${ }^{\ddagger}$ Cavendish Laboratory, University of Cambridge, JJ Thomson Avenue, Cambridge CB3 0HE, United Kingdom

${ }^{\S}$ Chemistry Research Laboratory, Mansfield Road, Oxford OX1 3TA, United Kingdom

"Computational Laboratory for Hybrid Organic Photovoltaics (CLHYO), CNR-ISTM, via Elce di Sotto 8, I-06123 Perugia, Italy

${ }^{\perp}$ Computation, Istituto Italiano di Tecnologia, Via Morego 30, 16163 Genova, Italy

Supporting Information

ABSTRACT: Solution-processable metal halide perovskites show immense promise for use in photovoltaics and other optoelectronic applications. The ability to tune their bandgap by alloying various halide anions (for example, in $\left.\mathrm{CH}_{3} \mathrm{NH}_{3} \mathrm{~Pb}\left(\mathrm{I}_{1-x} \mathrm{Br}_{x}\right)_{3}, 0<x<1\right)$ is however hampered by the reversible photoinduced formation of sub-bandgap emissive states. We find that ion segregation takes place via halide defects, resulting in iodide-rich low-bandgap regions close to the illuminated surface of the film. This segregation may be driven by the strong gradient in carrier generation rate through the thickness of these strongly absorbing materials. Once returned to the dark, entropically driven intermixing of halides returns the system to a homogeneous condition. We present approaches to suppress this process by controlling either the internal light distribution or the defect density within the film. These results are relevant to stability in both single- and mixed-halide perovskites, leading the way toward tunable and stable perovskite thin films for photovoltaic and light-emitting applications.
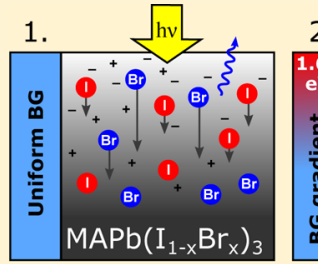

initial PL: $1.7-2.3 \mathrm{eV}$
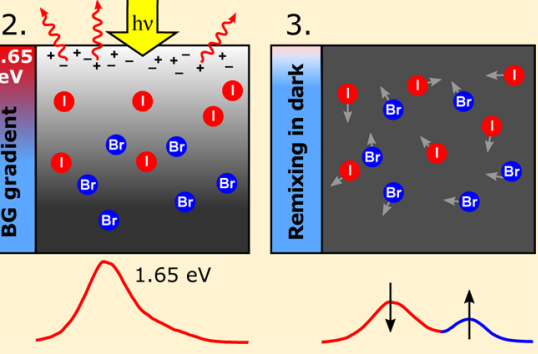

$\mathrm{L}$ ead halide perovskite solar cells have reached certified power conversion efficiencies (PCEs) exceeding 22\%. ${ }^{1}$ Additionally, these materials have been shown to demonstrate high photoluminescence quantum yields of more than $50 \%$ (at moderate carrier densities), ${ }^{2}$ which led to reports on optically pumped lasing structures and light-emitting devices (LEDs). ${ }^{3,4}$ The family of mixed-halide perovskites, $\mathrm{MAPb}$ $\left(\mathrm{I}_{1-x} \mathrm{Br}_{x}\right)_{3}\left(\mathrm{MA}=\mathrm{CH}_{3} \mathrm{NH}_{3}, 0 \leq x \leq 1\right)$, exhibits a continuously tunable bandgap from 1.6-2.3 eV simply by adjusting the ratio of halide precursors, ${ }^{5,6}$ with great appeal for application in multijunction solar cells and tunable LEDs. However, when mixed-halide perovskites are employed as solar cell absorber layers, the maximum attainable open-circuit voltage $\left(V_{\mathrm{OC}}\right)$ does not increase linearly with the change in the optical bandgap. A subsequent study by Hoke et al. $^{8}$ reported that white-light illumination induces formation of a strong and reversible subbandgap photoluminescence (PL) feature at around $1.68 \mathrm{eV}$, independent of the initial bandgap, which disappears after several minutes in the dark (hereafter referred to as the Hoke effect). That work, and subsequent others, ${ }^{9-11}$ attributed this behavior to phase separation and the formation of iodide-rich domains inducing low-bandgap states. Additionally, mixed halides using multiple A-site cations ${ }^{12,13}$ or nanocrystalline morphology ${ }^{14}$ display improved resilience to the Hoke effect, implying that the photoinduced changes are not necessarily an intrinsic phenomenon of these materials. However, the mechanisms driving segregation and remixing and the relative invariance of the sub-bandgap PL energy on initial material properties are not yet fully understood.

Understanding the origin of the Hoke effect and how to suppress it in operational devices is complicated by the many parameters on which it depends. The rate of growth of the sub-

Received: March 30, 2017

Accepted: May 11, 2017

Published: May 11, 2017 


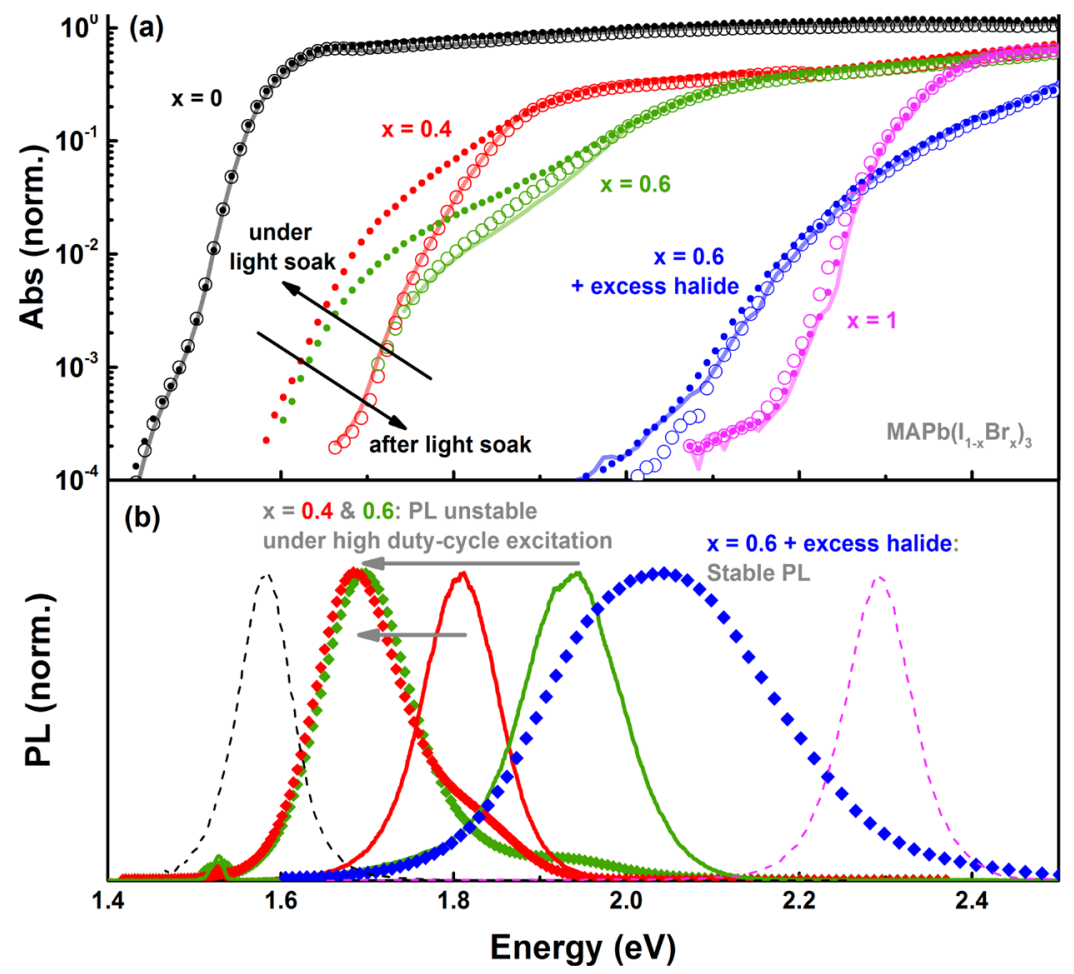

Figure 1. (a) PDS spectra for mixed-halide perovskite films, $\operatorname{MAPb}\left(\mathrm{I}_{1-x} \mathrm{Br}_{x}\right)_{3}$ measured before (lines), under (closed symbols), and after (open symbols) light soaking ( 0.2 sun). (b) PL spectra from excitation by low duty cycle (solid lines) or high duty cycle (diamonds) light pulses. PL spectra from single halide perovskites are shown with dashed lines for comparison.

bandgap PL has been shown to depend on the intensity and duty cycle of illumination, ${ }^{15,16}$ the history of sample exposure, ${ }^{17}$ the sample pressure, ${ }^{18}$ and the species of the A-site cation. ${ }^{12,13}$ We show that the effect also depends on the internal light distribution within the material, which is not well explained by existing models of halide segregation but is consistent with vertical halide segregation. We consider that ion segregation takes place via halide defects, resulting in iodide-rich lowbandgap regions close to the illuminated surface of the film. This segregation is driven by the strong gradient in carrier generation rate through the thickness of these strongly absorbing materials. Once returned to the dark, entropically driven intermixing of halides returns the system to a homogeneous condition.

Moreover, we demonstrate suppression of the Hoke effect in films engineered to experience a more homogeneous carrier generation profile and produce stable mixed-halide films by controlling the density of halide defects, pointing the way toward photovoltaic and optoelectronic devices with a tunable bandgap and stable $V_{\mathrm{oc}}$. This result is also relevant to singlehalide perovskites such as $\mathrm{MAPbI}_{3}$, which exhibit slow photoinduced changes in optical ${ }^{19}$ and electrical ${ }^{20,21}$ behavior associated with ion movement in the presence of charge carriers but without the strong photoluminescent indicator of ion migration that is exhibited by these mixed-halide thin films.

Photoinduced Sub-bandgap State Formation. We use linear absorption to study the effect of white light exposure on the optical properties of $\mathrm{MAPb}\left(\mathrm{I}_{1-x} \mathrm{Br}_{x}\right)_{3}$ films, where (unless otherwise stated) $x$ refers to the ratio of the halide in the perovskite precursor solutions. Figure 1a shows the photothermal deflection spectroscopy (PDS) spectra for iodidebromide perovskite samples $\mathrm{MAPb}\left(\mathrm{I}_{1-x} \mathrm{Br}_{x}\right)_{3}$ with $x$ ranging from 0 to 1 before (lines), under (closed symbols), and after (open symbols) light soaking with an intensity of $\sim 0.2$ sun (see Figure S1 for the white light LED spectrum). Measurements on pure tri-iodide $(x=0)$ and tribromide $(x=1)$ show no evident change upon light soaking. In contrast, exposing mixed-halide $(x=0.4-0.6)$ perovskite films to light soaking for $10 \mathrm{~min}$ generates a low-energy shoulder extending to $\sim 1.65 \mathrm{eV}$, independent of initial halide ratio. Upon removal of light soaking, these features quickly reduce in intensity, and within $10 \mathrm{~min}$, the absorption returns to the initial spectrum measured prior to light exposure. The absorbance levels of the defect shoulder (at $\sim 1.65 \mathrm{eV}$ ) under light soaking for the solid solutions are about $5 \%$ of the absorbance at the band edges for both the $x=0.4$ and 0.6 samples, indicating the formation of the observed sub-bandgap state in $\sim 5 \%$ of the total sample. Impedance spectra of devices made with these materials also show a photoinduced feature, which is suppressed at low temperatures (Figure S4). A third mixed-halide film with $x=$ 0.6 was prepared using a $1.7 \times$ excess of the halide-bearing precursor solutions. PDS of the excess-halide film shows a higher energy band edge to the stoichiometric $x=0.6$ film (likely due to to the preferential binding of $\mathrm{Pb}^{2+}$ and $\mathrm{Br}^{-}$ reported by Yoon et $\mathrm{al}^{22}$ ). More importantly, the absorption edge of the excess-halide film shows negligible change under light soaking.

It has been shown that the Hoke effect is more readily induced under continuous rather than pulsed illumination, ${ }^{16}$ implying that formation of the sub-bandgap state requires a sustained excited-state population. Thus, by exciting the mixedhalide samples with either low duty cycle $(3.1 \mathrm{eV}, 100 \mathrm{fs}, 1 \mathrm{kHz}$, $1 \mu \mathrm{J} \mathrm{cm}^{-2}$ pulse $\left.^{-1}\right)$ or high duty cycle $(3.05 \mathrm{eV}, 100 \mathrm{ps}, 40$ $\mathrm{MHz}, 46 \mathrm{pJ} \mathrm{\textrm {cm } ^ { - 2 }}$ pulse ${ }^{-1}$ ) light pulses, we collect the PL spectra associated with either the band-to-band or the photoinduced sub-bandgap emission, respectively. Figure $1 \mathrm{~b}$ 
shows that under high duty cycle illumination, PL from the $x=$ 0.4 and 0.6 samples is dominated by the sub-bandgap emission at $1.68 \mathrm{eV}$ associated with the low-energy shoulder in absorption measured under light soaking. In contrast, PL from the excess-halide sample was found to be stable under changes in illumination duty cycle, consistent with the stable absorption profile shown under light soaking.

Figure 2a shows the growth of the sub-bandgap PL feature under illumination from light sources with three different
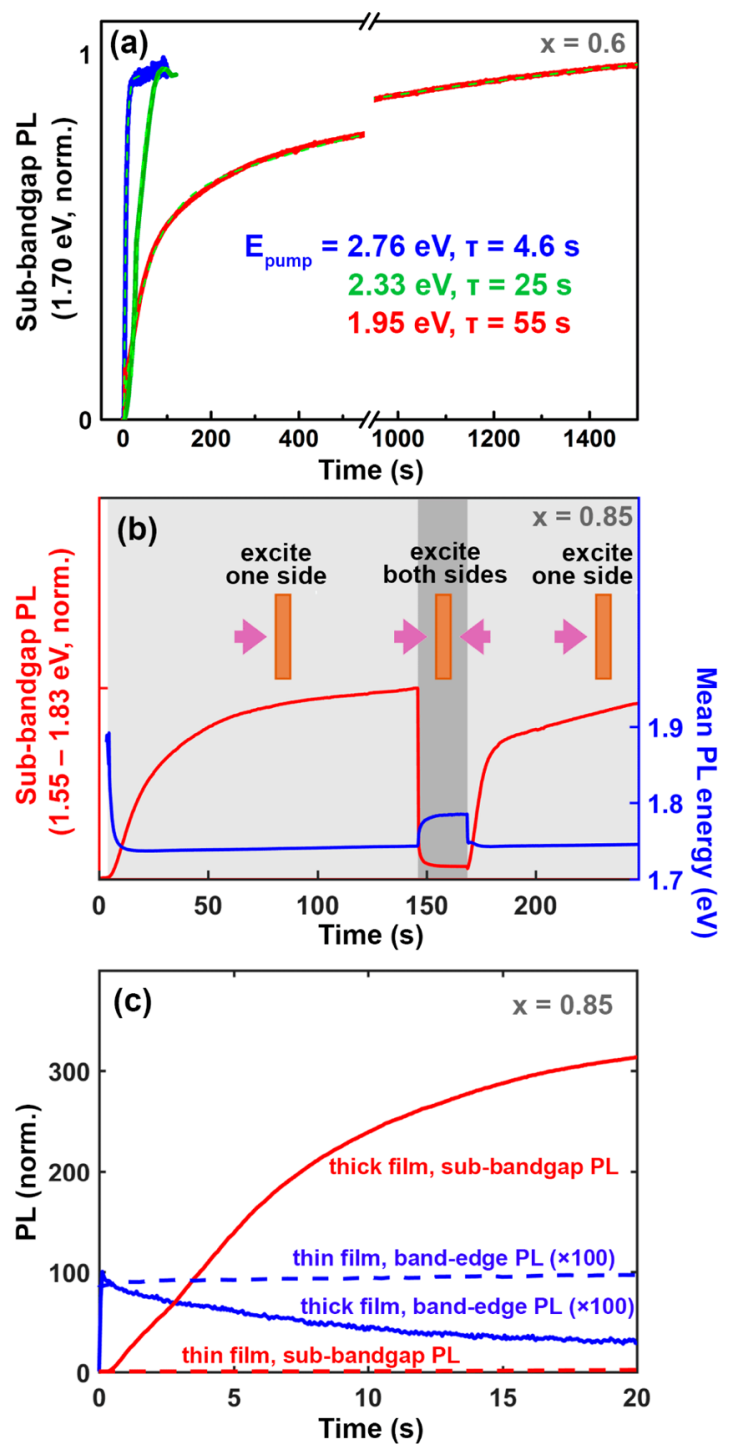

Figure 2. CW PL characterizations. (a) Excitation energy dependence of sub-bandgap PL growth of a $250 \mathrm{~nm}$ thick thin film using three different $\mathrm{CW}$ laser excitation sources with comparable absorbed photon flux. (b) Dependence of sub-bandgap PL growth and mean PL energy on excitation geometry. (c) Dependence of photoinduced changes in PL on film thickness.

wavelengths. The intensity of illumination was varied according to the absorption of the sample to ensure a similar flux of absorbed photons in each case (experimental parameters are given in Table S1). Under illumination at $2.76 \mathrm{eV}$, well above the bandgap, growth of the PL feature is well characterized by a monoexponential with time constant $\tau=4.6 \mathrm{~s}$. As the excess energy is reduced, the feature emerges progressively more slowly (even though the absorbed photon flux is higher), with $\tau$
$=25 \mathrm{~s}$ under $2.33 \mathrm{eV}$ illumination and $55 \mathrm{~s}$ when excited at 1.95 $\mathrm{eV}$, close to the optical bandgap. These changes in illumination wavelength determine not just the excess energy given to photogenerated charge carriers but also the internal distribution of carrier generation rate throughout the depth of the film as a result of the dependence of the absorption coefficient on wavelength. ${ }^{23}$ To decouple the role of hot carriers vs that of the internal light gradient, a beam splitter was used to provide excitation from either one of both sides of the sample (Figure 2b). After illumination from a single side had induced formation of the sub-bandgap feature, the addition of a second excitation source from the back of the sample resulted in quenching of $\mathrm{PL}$, even though the total intensity of excitation was approximately doubled $\left(3.05 \mathrm{eV}, 0.1 \mathrm{~W} \mathrm{~cm}^{-2}\right.$ from each side). This was accompanied by an increase of the mean PL energy, indicating a reduction in the efficiency of charge collection by iodide-rich regions under the more uniform charge generation profile.

If halide segregation requires a carrier generation gradient, the driving forces for segregation should be dependent on film opacity and therefore thickness. We thus prepared both thick $(280 \mathrm{~nm})$ and thin $(70 \mathrm{~nm})$ films of $\mathrm{MAPb}\left(\mathrm{I}_{1-x} \mathrm{Br}_{x}\right)_{3}$, with $x=$ 0.85 (absorption, PL, and SEM in Figures S16 and S17). Figure $2 \mathrm{c}$ shows the changes in band-edge and sub-bandgap PL upon continuous wave (CW) illumination $\left(3.05 \mathrm{eV}, 0.1 \mathrm{~W} \mathrm{~cm} \mathrm{~cm}^{-2}\right)$, normalized to the peak of the initial band-edge emission. While the difference in excitation conditions between the samples makes direct comparison difficult (the lower volume of the thinner film will result in higher carrier density, while an increased influence of surface traps may have the opposite effect), the pronounced differences in sub-bandgap PL behavior still merit consideration. Both the growth rate and final amplitude of the sub-bandgap PL are much higher in the thick film, which absorbs $>90 \%$ of the incident light. The thin film, which features the same absorption per unit thickness but only absorbs $\sim 40 \%$ of the total light (resulting in a lower variation of carrier generation rate across the film), displays a much slower rise and lower intensity of sub-bandgap PL, with negligible quenching of the high-energy PL from the pristine (nonsegregated) blend.

We now turn to structural and ultrafast optical analyses to understand the nature of the sub-bandgap states, the driving forces behind their formation (and relaxation), and why their emission energy is independent of the initial halide ratio.

Photophysics of the Photoinduced Sub-bandgap States. The physical processes underpinning the Hoke effect occur over two distinct time scales; light-induced halide segregation takes place over time scales of seconds, while the transport of photoinduced carriers from throughout the film to the iodide-rich (low-bandgap) regions occurs approximately 11 orders of magnitude more rapidly. In order to investigate both of these processes, we have conducted ultrafast transient absorption (TA) spectroscopy with and without light soaking. This pump-probe method is capable of tracking excited-state dynamics taking place in our samples between photoexcitation and PL.

Figure $3 \mathrm{a}$ presents the evolution of the differential transmission in the absence of light soaking. The short excitation pulses enabled investigation of excited-state processes without activating the sub-bandgap recombination pathway. Accordingly, the excited-state behavior closely resembles that of $\mathrm{MAPbI}_{3}$ and $\mathrm{MAPbBr}_{3}$ thin films (Figure S9). The signal is dominated by a ground-state bleach (GSB) due to occupation 

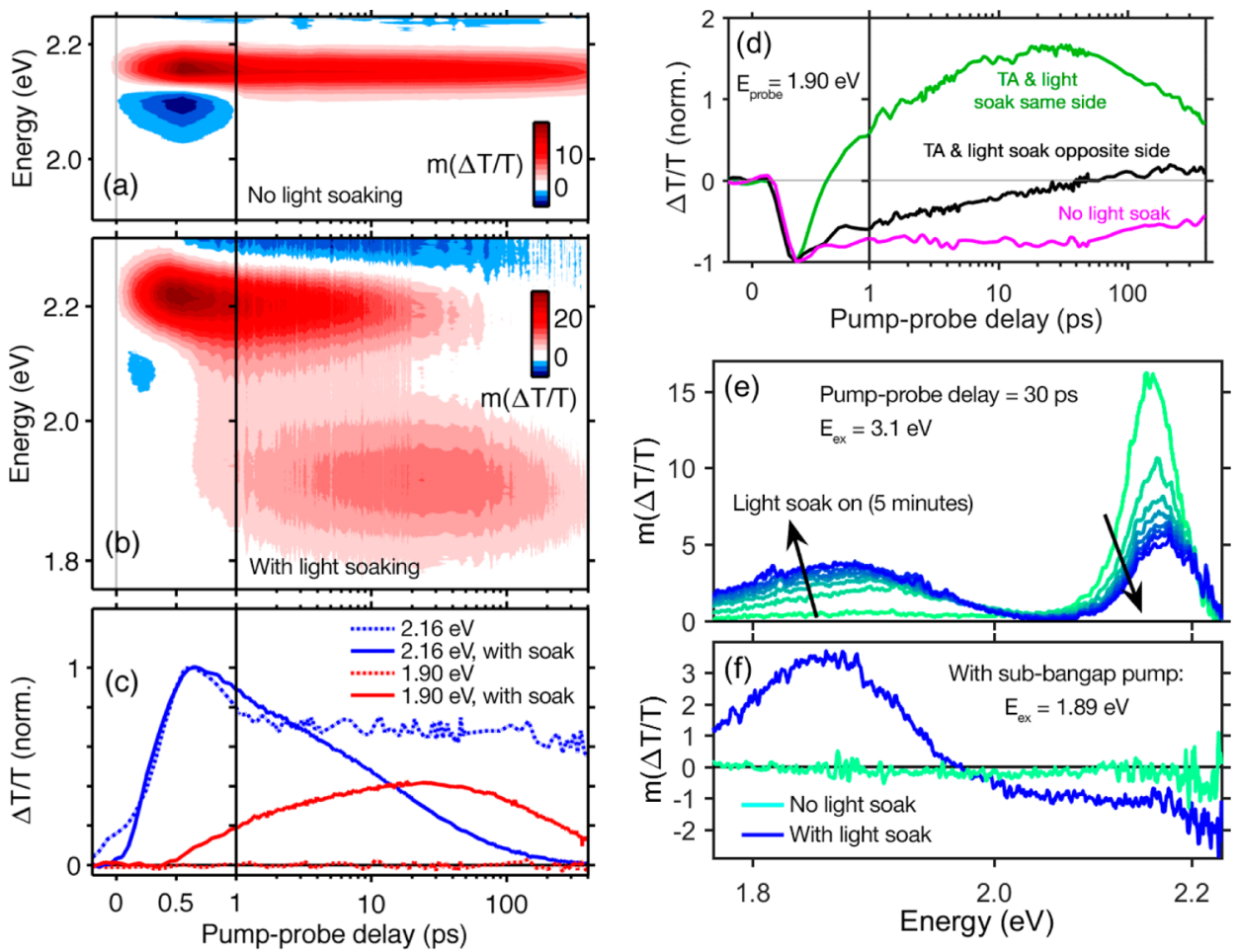

Figure 3. Ultrafast TA spectra of a $\operatorname{MAPb}\left(\mathrm{I}_{0.15} \mathrm{Br}_{0.85}\right)_{3}$ perovskite thin film excited at $3.1 \mathrm{eV}$, (a) without and (b) with constant light bias $(2.64$ $\mathrm{eV}, 0.3 \mathrm{~W} \mathrm{\textrm {cm } ^ { 2 }}$ ). The kinetics of the low- and high-energy bleach features are shown in (c) both with and without light soaking. (d) Kinetics of the low-energy bleach, with the light soaking and TA pulses illuminating either the same or opposite surfaces of the sample. (e) Spectral evolution of the signal at a pump-probe delay of 30 ps upon light soaking. (f) TA spectra at a pump-probe delay of 30 ps for excitation at $1.88 \mathrm{eV}$, below the optical gap of the main blend.

of excited states near the band edge (in this case, centered at around $2.16 \mathrm{eV}$, in agreement with absorption measurements), which can be taken as a measure for the photoinduced excitedstate density. ${ }^{2,24}$ Additional spectral features within the first 2 ps have been attributed to processes such as bandgap renormalization. ${ }^{25-27}$

Figure $3 \mathrm{~b}$ shows the TA map obtained when repeating the above experiment in the presence of above-gap light soaking $\left(2.64 \mathrm{eV}, 0.3 \mathrm{~W} \mathrm{~cm}^{-2}\right)$. Photoexcitation initially generates a single GSB as in Figure 3a. However, beginning at 0.5 ps, we see quenching of the main feature along with the concomitant emergence of a broad positive feature centered near $1.90 \mathrm{eV}$ and extending to at least $1.77 \mathrm{eV}$, similar to that previously reported by Yoon et al. ${ }^{17}$ No similar photoinduced changes in the TA spectra were observed in pure trihalide perovskite samples (Figure S9). We attribute this feature to the relaxation of carriers from the pristine semiconductor to the sub-bandgap states formed under CW light soaking. This process occurs extremely rapidly, with the sub-bandgap bleach rising to half of its maximum amplitude in $\sim 1$ ps. Under sufficient light soaking intensity, transfer to the sub-bandgap states can completely deplete the excited-state population in the pristine blend, a process that is complete within 100 ps for the data shown in Figure 3c. Unsurprisingly, the decay time of the quenched band-edge GSB is dependent on the intensity of light soaking. Figure S11 shows the GSB kinetics taken with a single TA pump power for a range of $\mathrm{CW}$ illumination intensities, with time constants decreasing from $91 \mathrm{ps}$ at $0.15 \mathrm{~W} \mathrm{~cm}^{-2}$ to $33 \mathrm{ps}$ at $0.9 \mathrm{~W} / \mathrm{cm}^{2}$. Such dependence could be the result of luminescent site density increasing under more intense light soaking; however, confocal mapping shows no indication of isolated iodide-rich sites on length scales larger than our spatial resolution $(<1 \mu \mathrm{m}$, Figure $\mathrm{S} 10)$. The faster GSB quenching may alternatively be due to an increasing energetic gradient that enhances carrier collection.

We studied the formation of sub-bandgap states by fixing the TA pump-probe delay at $30 \mathrm{ps}$ and observing the evolution of the spectral shape upon light soaking. As shown in Figure 3e, the sub-bandgap feature extends to lower energies as the photoinduced reorganization progresses and is mirrored by a blue shift of the above-gap feature, with both features becoming progressively broader. This behavior is consistent with photoinduced halide segregation, with progressive enrichment/depletion of bromide fraction (and therefore local bandgap) for the regions corresponding to the above/subbandgap TA features and a universal increase in energetic disorder. By reducing the energy of our pump pulses to $1.88 \mathrm{eV}$ (below the bandgap of the pristine material), we are able to exclusively probe the sub-bandgap states. Figure $3 \mathrm{f}$ shows TA spectra at a pump-probe delay of $10 \mathrm{ps}$. In the absence of light soaking, no excited-state features are observed. Upon illumination, we obtain the isolated signal from the subbandgap states with no contribution from the band edge, supporting our assignment of the photoinduced absorption in Figure 1 to the formation of the iodide-enriched material. The spectral shape strongly resembles that of a perovskite with a smaller bandgap than our $\mathrm{MAPb}\left(\mathrm{I}_{0.15} \mathrm{Br}_{0.85}\right)_{3}$ blend, including the band-edge bleach and broad negative feature above the bandgap. In contrast to the above-gap excitation, with a $1.88 \mathrm{eV}$ pump pulse, the sub-bandgap bleach forms within the instrument response time (Figure S12), indicating direct excitation of the sub-bandgap states. It is revealing that Figure 

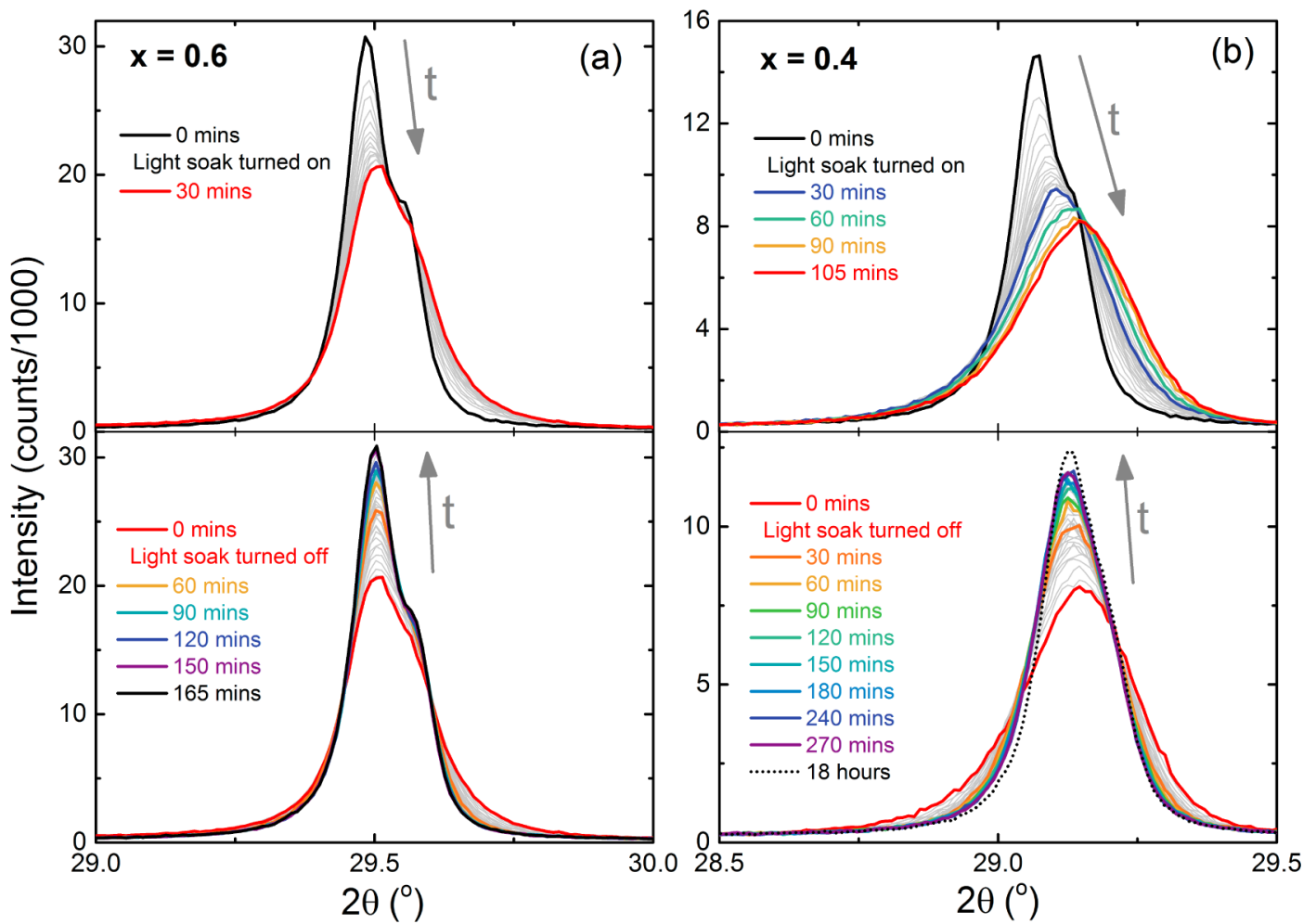

Figure 4. XRD patterns showing the time evolution of the (004) and (220) reflections for (a) $\mathrm{MAPb}\left(\mathrm{I}_{0.4} \mathrm{Br}_{0.6}\right)_{3}$ and $(\mathrm{b}) \mathrm{MAPb}\left(\mathrm{I}_{0.6} \mathrm{Br}_{0.4}\right)_{3}$ under light soaking (top) and after removal of light soaking (bottom).

$3 \mathrm{c}$ shows no direct excitation of the sub-bandgap states, consistent with the photoinduced regions of a low-bandgap perovskite comprising a very small fraction of the total sample but dominating the PL by collecting carriers prior to recombination.

Figure $3 a-c$ was collected with the pump, probe, and light soak beams in an approximately collinear geometry, with each of the light sources illuminating the same face of the sample. By rearranging the light soak laser to illuminate the rear face of the sample, we can track the relaxation of photoinduced carriers that are generated in the "dark" side of the film. Figure $3 \mathrm{~d}$ shows that carriers generated on the opposite side of the ( 280 $\mathrm{nm}$ thick) film take significantly longer (tens rather than several ps) to relax to sub-bandgap states and do so with a lower final yield. The increase in time required for carriers to experience and thermalize into low-bandgap states indicates that the photoinduced iodide-rich regions of the sample are located close to the illuminated surface, where carrier generation rate is the highest.

Photoinduced Structural Modifications: XRD Studies. We investigate the structural origin of the changes in the photophysical properties of mixed-halide perovskites by analyzing the light-soaking-induced changes in the crystal lattice with $\mathrm{X}$-ray diffraction (XRD) measurements on thin spin-coated $\mathrm{MAPb}\left(\mathrm{I}_{1-x} \mathrm{Br}_{x}\right)_{3}$ films $(d \approx 200 \mathrm{~nm})$. In contrast to the report of Hoke et al., ${ }^{8}$ no new peaks were observed to form under light soaking in any of the films studied. Figure 4 shows the XRD pattern for the (400) and (220) reflections of $x=0.6$ and 0.4 perovskites as a function of time, upon application and following removal of $\mathrm{CW}$ light soaking. In both the presence and absence of light soaking, the changes in $\mathrm{MAPb}\left(\mathrm{I}_{0.6} \mathrm{Br}_{0.4}\right)_{3}$ are observed to occur over a longer time period than those in
$\operatorname{MAPb}\left(\mathrm{I}_{0.4} \mathrm{Br}_{0.6}\right)_{3}$. This is consistent with observations of faster sub-bandgap PL growth rates with increasing bromide fraction. ${ }^{8}$

With light soaking, we observe a shift of the peaks to higher $2 \theta$ due to a reduction in the unit cell volume. This is accompanied by a reduction in the intensity and a broadening of the reflections. Upon removal of light soaking, the initial peak intensity and shape are recovered, with no change in the scattering angle, $2 \theta$. Given the reversibility of the peak broadening, we attribute this to bulk strain rather than a reduction in crystallite size within the films.

A Le Bail analysis ${ }^{28}$ of the XRD data (Table S2) reveals that the photoinduced changes in the structure of the mixed-halide perovskites can be separated into three distinct regimes: (1) the initial structure prior to light soaking; (2) (during soaking) a modified bulk structure with reduced volume, increased tetragonal distortion, and increased strain; and (3) (after light soaking is removed) a reduced volume phase with a small tetragonal distortion and low strain. The origin of the photoinduced changes in the XRD can thus be ascribed to at least two separate phenomena. First is a reversible component arising from changes in the tetragonal distortion and strain that is associated with the reversible ion segregation and subbandgap PL. Second is an irreversible contraction of the unit cell volume upon initial light soaking, which could be a result of irreversible defect migration (such as the annihilation of halide vacancies and interstitials ${ }^{29}$ ).

XRD measurements of pure tribromide and tri-iodide perovskites did not show any changes to either the peak position or width (Figure S7). Additionally, temperaturedependent XRD measurements did not replicate the above phenomena in any of the films (Figure S14), indicating that the photoinduced changes are due to the presence of charge carriers rather than localized sample heating. 

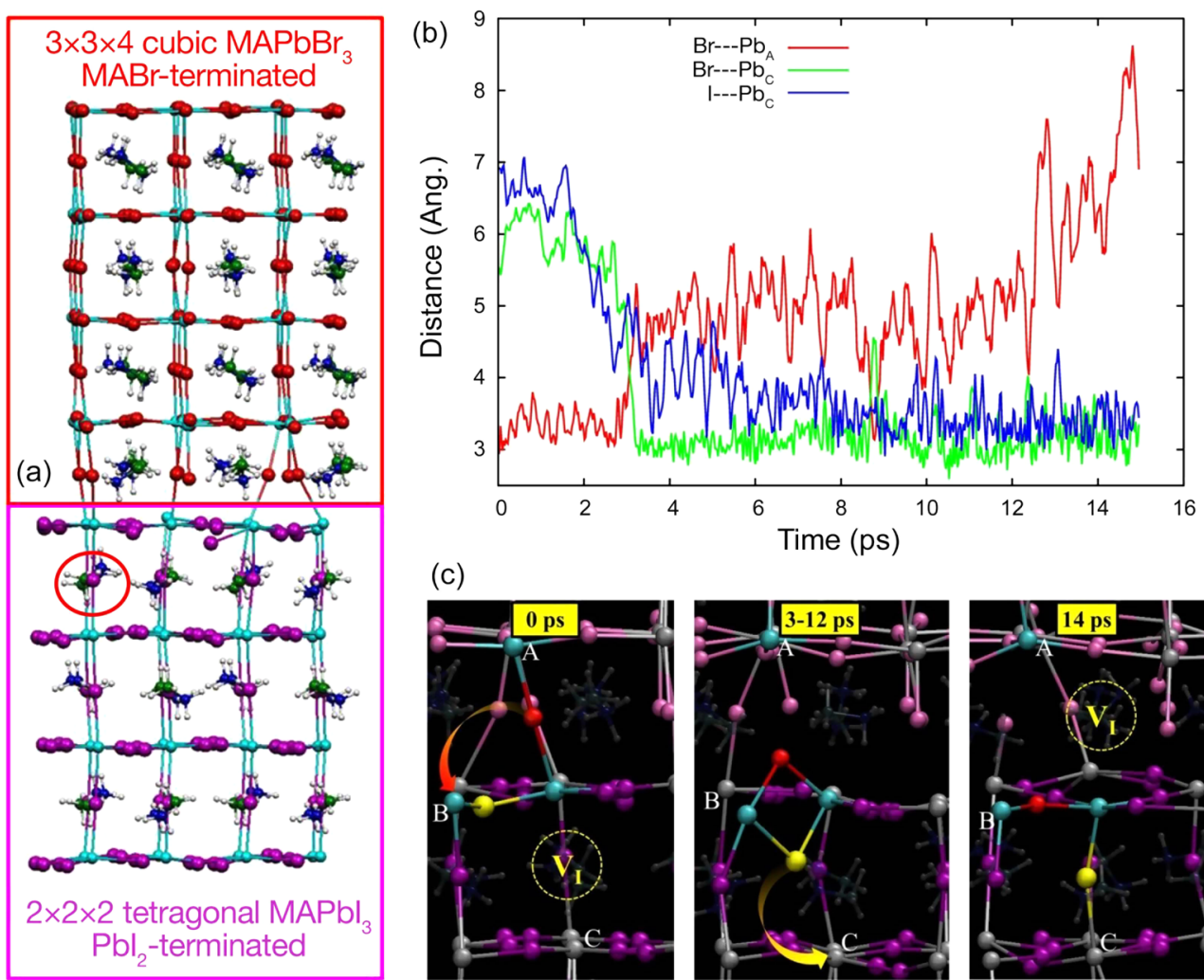

(c)

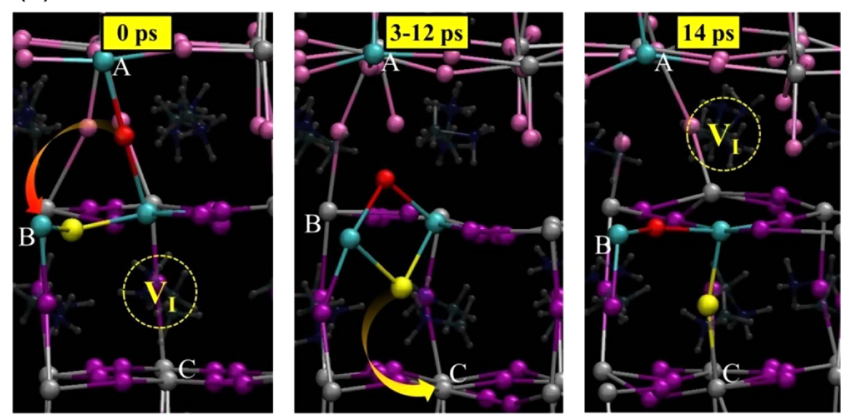

Figure 5. (a) Model of the $\mathrm{MAPbBr}_{3} / \mathrm{MAPbI}_{3}$ interface. The red circle in $\mathrm{MAPbI}_{3}$ indicates the site where an iodide vacancy is created. (b) Time evolution of the $\mathrm{Pb}-\mathrm{Br}$ and $\mathrm{Pb}-\mathrm{I}$ distances (see labels in the left panel of Figure 3c). (c) Structural evolution following defect migration. At time $=0$, an iodide vacancy is present in $\mathrm{MAPbI}_{3}$. At $t=3 \mathrm{ps}$, a mixed $\mathrm{I}-\mathrm{Br}$ split interstitial is formed, which is stable up to $t=$ $12 \mathrm{ps}$. Afterward $(t=14 \mathrm{ps})$, the iodide vacancy is transferred to the $\mathrm{MAPbBr}_{3}$ phase.

Halide Transport and the Stability of Intermixed vs Segregated Configurations. To investigate the electronic properties of the $\mathrm{MAPbBr} 3 / \mathrm{MAPbI}_{3}$ assembly and the possible ion migration across this interface, we have built a model based on the best lattice matching between cubic $\mathrm{MAPbBr}_{3}$ and tetragonal $\mathrm{MAPbI}_{3}$. The employed model, reported in Figure 5a (details in the SI), is based on a MABr-terminated $3 \times 3 \times 4$ cubic $\mathrm{MAPbBr}_{3}$ slab interfaced with a $\mathrm{PbI}_{2}$-terminated $2 \times 2 \times 4$ tetragonal $\mathrm{MAPbI}_{3}$ slab. Such a combination allows us to use the $\mathrm{MAPbI}_{3}$ experimental cell parameters with a lattice mismatch with respect to $\mathrm{MAPbBr}_{3}$ within $0.1 \%$. The employed model shows nine terminal $\mathrm{Br}$ atoms (from $\mathrm{MAPbBr}_{3}$ ) interfaced with eight surface $\mathrm{Pb}$ atoms (from $\mathrm{MAPbI}_{3}$ ). Upon geometry optimization, a total of seven $\mathrm{Br}-\mathrm{Pb}$ interfacial bonds are created, thus leading to an almost perfect saturation of undercoordinated interface atoms.

To investigate the possible ion migration across the $\mathrm{MAPbBr} 3 / \mathrm{MAPbI}_{3}$ interface, we subjected the model system of Figure $5 \mathrm{a}$ to $\mathrm{ab}$ initio molecular dynamics simulations; we heated up the system to $450 \mathrm{~K}$ and let it evolve in time for 20 ps after a few ps of thermalization. During this time, we monitored the evolution of the interfacial bond lengths but did not observe any ion migration, which is quite unlikely on the observed temperature and time range in an almost perfectly saturated system. The calculated activation energy for exchange of an iodide atom in bulk $\mathrm{MAPbI}_{3}$ is $\sim 0.5 \mathrm{eV}$ (this process corresponds to $90^{\circ}$ rotation of one $\mathrm{PbI}_{6}$ octahedron along the $c$ axis). This barrier is too high to be overcome during our relatively short dynamics simulations (on ps time scales) and is 1 order of magnitude higher than the activation energy associated with the photoinduced feature shown in impedance spectroscopy of mixed halides (Figure S4).

We thus created an iodide vacancy in the second $\mathrm{MAPbI}_{3}$ layer away from the interface (Figure 5a) and repeated the $a b$ initio molecular dynamics simulations. By following the time evolution of the relevant geometrical parameters (Figure $5 b$ ), we observed migration of an interfacial $\mathrm{Br}^{-}$ion within $\sim 3 \mathrm{ps}$, as testified by the sudden increase of the $\mathrm{Pb}-\mathrm{Br}$ distance corresponding to the initially bound couple (Figure $5 b$ ). Following this migration, a mixed $\mathrm{I}^{-} / \mathrm{Br}^{-}$split interstitial defect is created, leaving behind a $\mathrm{Br}^{-}$vacancy in $\mathrm{MAPbBr}_{3}$, which is stable for about 10 ps (3-12 ps range in Figure 5c). After this time, another migration event occurs, leading to incorporation of $\mathrm{Br}^{-}$into the $\mathrm{MAPbI}_{3}$ lattice and creation of a $\mathrm{MAPbBr}_{3}$ bromide vacancy (14 ps range in Figure $5 \mathrm{c}$ ). The driving force for defect migration is probably the slight halide imbalance at the interface ( $9 \mathrm{Br}$ atoms that bind to $8 \mathrm{~Pb}$ centers), but we cannot exclude $\mathrm{Br}^{-}$vacancy stabilization compared to formation of an $\mathrm{I}^{-}$vacancy. The observed relatively short time for vacancy migration is consistent with the recently reported activation energies for such processes, which amounts to $\sim 0.1 \mathrm{eV}$ for $\mathrm{MAPbI}_{3}$, with bromide vacancy migration in $\mathrm{MAPbBr}_{3}$ featuring a reduced activation energy. ${ }^{30,31}$

To check whether a halide-segregated phase could be stabilized by light absorption with respect to a dispersed phase in the absence of defects, we considered two bulk systems with the same $3: 1 \mathrm{I} / \mathrm{Br}$ stoichiometry but with bromide either evenly dispersed or concentrated in a specific region (Figure S19). To approximately simulate the effect of light absorption, we considered here the lowest triplet excited state 
of both systems. Notably, in both the ground and excited states, the Br-dispersed phase is more stable than the $\mathrm{Br}$-concentrated phase by 0.13 and $0.06 \mathrm{eV}$, respectively. Thus, our simulations performed on perfectly crystalline bulk phases do show an energy gain associated with halide segregation, although the driving force for this process is not sufficient alone to stabilize the phase-segregated phase.

Overall, our computational analyses suggest that halide defects, typically halide vacancies and interstitials, promote halide migration by providing low-energy migration pathways. Notably, the defect-assisted halide migration in $\mathrm{MAPbI}_{3}$ was proposed to be activated by light, ${ }^{28}$ in line with the present observations.

Photoinduced Halide Segregation and Methods of Passivation. Recent investigations of the mechanics of the Hoke effect have ascribed its origin to polaron formation or a miscibility gap of the solid mixed-halide solution due to changes in the Helmholz free energy upon photoexcitation..$^{9,11,15}$ While our calculations do indicate partial stabilization of the halide-segregated configuration under illumination, the existing models do not explain our observed dependence of the Hoke effect on illumination wavelength, excitation geometry, and film thickness. These effects imply the role of an internal gradient in light intensity (and therefore carrier generation rate) in driving halide segregation; therefore, we consider here a mechanism by which such a gradient may contribute to the Hoke effect.

The internal gradient in carrier generation rate may induce ion migration via the photoinduced strain gradient in the lattice indicated in our XRD results or, more directly, for example, with the excess energy from hot carriers thermalizng near the illuminated surface, providing energy to activate halide defects (with calculated values of $50 \mathrm{meV}$ deposited from carriers to each molecule at the surface of a film under solar illumination $^{32}$ ). We do not consider that the effect is due to bulk heating of the sample; experiments that applied a temperature gradient across the sample were unable to induce halide segregation (Figures S14 and S15).

Results in the literature support the role of light gradients in affecting ion migration; deQuilletes et al. have demonstrated iodide migration away from illuminated regions in $\mathrm{MAPbI}_{3}$, and ion drift velocities in thin films of $\mathrm{MAPbI}_{3}$ have been shown to increase substantially under illumination. ${ }^{33}$ If halide anions are more mobile near the illuminated surface, this would result in net transport down into the film, migrating via halide defect sites. In a mixed halide, any difference in hopping rate between bromide and iodide would result in a vertical gradient of the iodide/bromide ratio and therefore of local bandgap. Carriers generated in the resulting material will be funnelled to the lowest-energy region, ${ }^{34,35}$ recombining to generate the subbandgap PL characteristic of the Hoke effect. The slower relaxation of carriers generated opposite the illuminated face in Figure $3 \mathrm{~d}$ reveals that the iodide-rich low-bandgap region is located at the illuminated surface of the film, corresponding to faster migration of bromide in the vertical segregation model.

Once carriers are funnelled to the low-bandgap regions, the rate of nonradiative recombination is lower due to local enhancement of carrier density, which fills traps and increases bimolecular recombination, explaining the much higher intensity of the sub-bandgap PL compared with the pristine blend. Specifically, although PDS (Figure 1a) shows that subbandgap regions comprise $<5 \%$ of sample volume, they can fully capture all excitations in less than the radiative lifetime
(Figure 3c), implying at least a 20 -fold increase in local carrier density.

When CW illumination (or the internal light gradient, as in Figure 2b) is removed, the vertical gradient in carrier generation rate disappears and the sample undergoes remixing to the homogeneous state, albeit at a slower rate, ${ }^{17}$ possibly because ion mobilities are reduced in the dark. ${ }^{33}$ Our calculations show that this intermixing is energetically favored by $0.13 \mathrm{eV}$. This may work in tandem with the proposed driving force for remixing due to differences in the free energy of mixing in the presence of carriers. ${ }^{11}$

Our computational investigations show that ion migration in mixed-halide systems takes place via halide defects. Consistently, Figure 1 shows that a film prepared with a large excess of the halide-bearing precursors does not exhibit the Hoke effect. The abundance of halide ions available during film crystallization should result in a film with reduced halide vacancies, thus lowering ion mobility. Furthermore, both the absorption and PL show higher disorder than the stoichiometric films, which we attribute to spatial variations in halide ratio being "frozen in" during film formation. The low density of halide vacancies would preclude the entropy-driven halide intermixing process that takes place in the dark and that is likely the origin of improvements in film homogeneity that have been observed to take place after initial film preparation in stoichiometric mixed-halide films. ${ }^{6}$

These results explain the absence of photoinduced subbandgap states in mixed-halide nanocrystals, ${ }^{14}$ which benefit doubly, having both low halide defect density and negligible difference in carrier generation rate across a given crystal.

The final piece of this puzzle is the independence of the subbandgap PL energy on the initial halide ratio, sample preparation, and light intensity. This energy, $1.68 \mathrm{eV}$, corresponds to a mixed-halide sample with a composition of $x \approx 0.20 .^{36}$ This value is coincident with a tetragonal-to-cubic phase transition reported by Noh and co-workers ${ }^{5}$ as lying between $x=0.13$ and 0.2 . It is possible that bromide depletion at the illuminated surface proceeds until $x \approx 0.20$, beyond which the lattice phase transition presents an energetic barrier to further depletion, determining the final energy of the subbandgap emission. Indeed, the existence of a stable alloy at room temperature with ratio $x<0.19$ has been predicted from thermodynamic analysis ${ }^{9}$ and is consistent with ratios that have been empirically determined to provide stable performance when used in photovoltaic devices. ${ }^{5,37}$

It is important to note that many of the factors governing the strength of the Hoke effect, such as illumination intensity, sample history, and use of an excess of halide precursor or mixed A-site cations, have also been shown to correlate with the stability of the intensely studied tri-iodide perovskites, in particular, with the degree of hysteresis observed in device performance.

Confidence in the attribution of both the Hoke effect and hysteresis in trihalide devices to ion migration continues to grow with the rapid rate of publication on these materials. Indeed, it is possible that the underlying processes are due to similar mechanisms and that the presence of two mobile anions with different mobilities along with the strong dependence of material bandgap on local halide ratio simply provides a sensitive photoluminescent tag revealing ion migration in mixed-halide perovskites. For these reasons, understanding (and controlling) the Hoke effect may guide improvements in 
the stability not just of tunable and high-bandgap materials but of metal halide perovskites in general.

In summary, our results on the structural and photophysical properties of mixed lead halide perovskites are in agreement with defect-assisted migration of halide ions away from the film surface. It is possible that this migration is a result of the steep carrier generation gradient that exists during illumination. In this model, the slower hopping rate of iodide results in bromide depletion to a final composition of $\mathrm{MAPb}\left(\mathrm{I}_{0.8} \mathrm{Br}_{0.2}\right)_{3}$, where a cubic-to-tetragonal phase transition prevents further segregation, determining the $1.68 \mathrm{eV}$ energy of the final photoinduced sub-bandgap emission.

The ability to control or prevent photoinduced halide segregation will be crucial to realizing tunable mixed-halide optoelectronic devices. We have demonstrated both structural and compositional approaches using film thickness and precursor ratio to alter the susceptibility of mixed-halide films to ion segregation. In addition, the use of colloidal perovskite crystals, which exhibit both low defect density and low carrier generation inhomogeneity, may provide a useful avenue for the creation of stable tunable devices. Indeed, sufficient control of spatial differences in halide ratio may even enable the creation of multilevel systems for applications such as lasing, as has been demonstrated recently ${ }^{38}$ by exploiting temperature-dependent phase transitions in $\mathrm{MAPbI}_{3}$. Furthermore, mixed-halide systems (and the stabilization methods presented here) have direct relevance to the more intensely studied family of trihalide perovskites (such as $\mathrm{MAPbI}_{3}$ ), which feature the same photoinduced halide migration processes but lack the clear photoluminescent indicator of migration described in this work.

\section{ASSOCIATED CONTENT}

\section{S Supporting Information}

The Supporting Information is available free of charge on the ACS Publications website at DOI: 10.1021/acsenergylett.7b00282.

Experimental and computational methods, Le Bail analysis of XRD, temperature-dependent impedance spectroscopy, and additional structural (XRD, WAXS, GIWAXS) and optical (ultrafast TA, PL, confocal PL mapping) characterization of mixed- and trihalide samples including at low temperature, with applied heat gradients, and of cesium lead mixed-halide perovskite (PDF)

\section{AUTHOR INFORMATION}

\section{Corresponding Authors}

*E-mail: alex.barker@iit.it (A.J.B.).

*E-mail: as2233@cam.ac.uk (A.S.).

*E-mail: annamaria.petrozza@iit.it (A.P.).

*E-mail: rhf10@cam.ac.uk (R.H.F.).

\section{ORCID}

Alex J. Barker: 0000-0002-5614-4113

Aditya Sadhanala: 0000-0003-2832-4894

Filippo De Angelis: 0000-0003-3833-1975

Annamaria Petrozza: 0000-0001-6914-4537

\section{Author Contributions}

${ }^{\#}$ A.J.B. and A.S. contributed equally.

\section{Notes}

The authors declare no competing financial interest.

\section{ACKNOWLEDGMENTS}

We acknowledge funding from the Engineering and Physical Sciences Research Council (EPSRC) and the Winton Programme (Cambridge) for the Physics of Sustainability. The research leading to these results has received funding from the European Union Seventh Framework Programme (FP7/ 2007-2013) under Grant Agreement No. 604032 of the MESO project. A.S. acknowledges funding and support from the Indo-UK APEX project. F.D. acknowledges funding from Herchel Smith fellowship. S.P.S. acknowledges funding from the Royal Society for the Newton International Fellowship. A.J.P. thanks David Lidzey (University of Sheffield) for use of a sample chamber for GIWAXS measurements. We thank Diamond Light Source for access to beamlines I12 and I07 (sessions EE11454-1 and SI12436-1, respectively) that contributed to the results presented here. We thank Prof. Paul Bristowe, Prof. Aron Walsh, Dr. Jung-Hoon Lee, and Dr. James Ball for helpful discussions.

\section{REFERENCES}

(1) NREL. Best Research-Cell Efficiencies. https://www.nrel.gov/pv/ assets/images/efficiency-chart.png (accessed April 1, 2017).

(2) Deschler, F.; Price, M.; Pathak, S.; Klintberg, L. E.; Jarausch, D.D.; Higler, R.; Hüttner, S.; Leijtens, T.; Stranks, S. D.; Snaith, H. J.; et al. High Photoluminescence Efficiency and Optically Pumped Lasing in Solution-Processed Mixed Halide Perovskite Semiconductors. J. Phys. Chem. Lett. 2014, 5, 1421-1426.

(3) Cho, H.; Jeong, S.-H.; Park, M.-H.; Kim, Y.-H.; Wolf, C.; Lee, C.L.; Heo, J. H.; Sadhanala, A.; Myoung, N.; Yoo, S.; et al. Overcoming the electroluminescence efficiency limitations of perovskite lightemitting diodes. Science 2015, 350, 1222-1225.

(4) Sadhanala, A.; Ahmad, S.; Zhao, B.; Giesbrecht, N.; Pearce, P. M.; Deschler, F.; Hoye, R. L. Z.; Gödel, K. C.; Bein, T.; Docampo, P.; et al. Blue-Green Color Tunable Solution Processable Organolead Chloride-Bromide Mixed Halide Perovskites for Optoelectronic Applications. Nano Lett. 2015, 15, 6095-6101.

(5) Noh, J. H.; Im, S. H.; Heo, J. H.; Mandal, T. N.; Seok, S. Il. Chemical Management for Colorful, Efficient, and Stable InorganicOrganic Hybrid Nanostructured Solar Cells. Nano Lett. 2013, 13, 1764-1769.

(6) Sadhanala, A.; Deschler, F.; Thomas, T. H.; Dutton, S. E.; Goedel, K. C.; Hanusch, F. C.; Lai, M. L.; Steiner, U.; Bein, T.; Docampo, P.; et al. Preparation of Single-Phase Films of $\mathrm{CH}_{3} \mathrm{NH}_{3} \mathrm{~Pb}-$ $\left(\mathrm{I}_{1-\mathrm{x}} \mathrm{Br}_{\mathrm{x}}\right)_{3}$ with Sharp Optical Band Edges. J. Phys. Chem. Lett. 2014, 5, $2501-2505$.

(7) Gil-Escrig, L.; Miquel-Sempere, A.; Sessolo, M.; Bolink, H. J. Mixed Iodide-Bromide Methylammonium Lead Perovskite-based Diodes for Light Emission and Photovoltaics. J. Phys. Chem. Lett. 2015, 6, 3743-3748.

(8) Hoke, E. T.; Slotcavage, D. J.; Dohner, E. R.; Bowring, A. R; Karunadasa, H. I.; McGehee, M. D. Reversible photo-induced trap formation in mixed-halide hybrid perovskites for photovoltaics. Chem. Sci. 2015, 6, 613-617.

(9) Brivio, F.; Caetano, C.; Walsh, A. Thermodynamic Origin of Photoinstability in the $\mathrm{CH}_{3} \mathrm{NH}_{3} \mathrm{~Pb}\left(\mathrm{I}_{1-\mathrm{x}} \mathrm{Br}_{\mathrm{x}}\right)_{3}$ Hybrid Halide Perovskite Alloy. J. Phys. Chem. Lett. 2016, 7, 1083-1087.

(10) Slotcavage, D. J.; Karunadasa, H. I.; McGehee, M. D. LightInduced Phase Segregation in Halide-Perovskite Absorbers. ACS Energy Lett. 2016, 1, 1199-1205.

(11) Bischak, C. G.; Hetherington, C. L.; Wu, H.; Aloni, S.; Ogletree, D. F.; Limmer, D. T.; Ginsberg, N. S. Origin of Reversible Photoinduced Phase Separation in Hybrid Perovskites. Nano Lett. 2017, 17, 1028-1033.

(12) McMeekin, D. P.; Sadoughi, G.; Rehman, W.; Eperon, G. E.; Saliba, M.; Horantner, M. T.; Haghighirad, A.; Sakai, N.; Korte, L.; Rech, B.; et al. A mixed-cation lead mixed-halide perovskite absorber for tandem solar cells. Science 2016, 351, 151-155. 
(13) Beal, R. E.; Slotcavage, D. J.; Leijtens, T.; Bowring, A. R.; Belisle, R. A.; Nguyen, W. H.; Burkhard, G.; Hoke, E. T.; McGehee, M. D. Cesium lead halide perovskites with improved stability for tandem solar cells. J. Phys. Chem. Lett. 2016, 7, 746-751.

(14) Akkerman, Q. A.; D’Innocenzo, V.; Accornero, S.; Scarpellini, A.; Petrozza, A.; Prato, M.; Manna, L. Tuning the Optical Properties of Cesium Lead Halide Perovskite Nanocrystals by Anion Exchange Reactions. J. Am. Chem. Soc. 2015, 137, 10276-10281.

(15) Slotcavage, D. J.; Karunadasa, H. I.; McGehee, M. D. LightInduced Phase Segregation in Halide-Perovskite Absorbers. ACS Energy Lett. 2016, 1, 1199-1205.

(16) Yang, X.; Yan, X.; Wang, W.; Zhu, X.; Li, H.; Ma, W.; Sheng, C. Light induced metastable modification of optical properties in $\mathrm{CH}_{3} \mathrm{NH}_{3} \mathrm{PbI}_{3-\mathrm{x}} \mathrm{Br}_{\mathrm{x}}$ perovskite films: Two-step mechanism. Org. Electron. 2016, 34, 79-83.

(17) Yoon, S. J.; Draguta, S.; Manser, J. S.; Sharia, O.; Schneider, W. F.; Kuno, M.; Kamat, P. V. Tracking Iodide and Bromide Ion Segregation in Mixed Halide Lead Perovskites during Photoirradiation. ACS Energy Lett. 2016, 1, 290-296.

(18) Jaffe, A.; Lin, Y.; Beavers, C. M.; Voss, J.; Mao, W. L.; Karunadasa, H. I. High-Pressure Single-Crystal Structures of 3D LeadHalide Hybrid Perovskites and Pressure Effects on their Electronic and Optical Properties. ACS Cent. Sci. 2016, 2, 201-209.

(19) Tian, Y.; Peter, M.; Unger, E.; Abdellah, M.; Zheng, K.; Pullerits, T.; Yartsev, A.; Sundström, V.; Scheblykin, I. G. Mechanistic insights into perovskite photoluminescence enhancement: light curing with oxygen can boost yield thousandfold. Phys. Chem. Chem. Phys. 2015, $17,24978-24987$.

(20) Snaith, H. J.; Abate, A.; Ball, J. M.; Eperon, G. E.; Leijtens, T.; Noel, N. K.; Stranks, S. D.; Wang, J. T.-W.; Wojciechowski, K.; Zhang, W. Anomalous Hysteresis in Perovskite Solar Cells. J. Phys. Chem. Lett. 2014, 5, 1511-1515.

(21) Leijtens, T.; Stranks, S. D.; Eperon, G. E.; Lindblad, R.; Johansson, E. M. J.; McPherson, I. J.; Rensmo, H.; Ball, J. M.; Lee, M. M.; Snaith, H. J. Electronic Properties of Meso-Superstructured and Planar Organometal Halide Perovskite Films: Charge Trapping, Photodoping, and Carrier Mobility. ACS Nano 2014, 8, 7147-7155.

(22) Yoon, S.; Stamplecoskie, K. G.; Kamat, P. V. How Lead Halide Complex Chemistry Dictates the Composition of Mixed Halide Perovskites. J. Phys. Chem. Lett. 2016, 7, 1368-1373.

(23) Lin, Q.; Armin, A.; Nagiri, R. C. R.; Burn, P. L.; Meredith, P. Electro-optics of perovskite solar cells. Nat. Photonics 2014, 9, 106112.

(24) Manser, J. S.; Kamat, P. V. Band filling with free charge carriers in organometal halide perovskites. Nat. Photonics 2014, 8, 737-743.

(25) Grancini, G.; Srimath Kandada, A. R.; Frost, J. M.; Barker, A. J.; De Bastiani, M.; Gandini, M.; Marras, S.; Lanzani, G.; Walsh, A.; Petrozza, A. Role of microstructure in the electron-hole interaction of hybrid lead halide perovskites. Nat. Photonics 2015, 9, 695-701.

(26) Price, M. B.; Butkus, J.; Jellicoe, T. C.; Sadhanala, A.; Briane, A.; Halpert, J. E.; Broch, K.; Hodgkiss, J. M.; Friend, R. H.; Deschler, F. Hot-carrier cooling and photoinduced refractive index changes in organic-inorganic lead halide perovskites. Nat. Commun. 2015, 6, 8420.

(27) Chen, K.; Barker, A. J.; Morgan, F. L. C.; Halpert, J. E.; Hodgkiss, J. M. Effect of Carrier Thermalization Dynamics on Light Emission and Amplification in Organometal Halide Perovskites. J. Phys. Chem. Lett. 2015, 6, 153-158.

(28) Le Bail, A.; Duroy, H.; Fourquet, J. L. Ab-initio structure determination of LiSbWO6 by X-ray powder diffraction. Mater. Res. Bull. 1988, 23, 447-452.

(29) Mosconi, E.; Meggiolaro, D.; Snaith, H. J.; Stranks, S. D.; De Angelis, F. Light-induced annihilation of Frenkel defects in organolead halide perovskites. Energy Environ. Sci. 2016, 9, 3180-3187.

(30) Azpiroz, J. M.; Mosconi, E.; Bisquert, J.; De Angelis, F. Defect migration in methylammonium lead iodide and its role in perovskite solar cell operation. Energy Environ. Sci. 2015, 8, 2118-2127.

(31) Meloni, S.; Moehl, T.; Tress, W.; Franckevičius, M.; Saliba, M.; Lee, Y. H.; Gao, P.; Nazeeruddin, M. K.; Zakeeruddin, S. M.;
Rothlisberger, $\mathrm{U}_{\text {; }}$ et al. Ionic polarization-induced current-voltage hysteresis in $\mathrm{CH}_{3} \mathrm{NH}_{3} \mathrm{PbX}_{3}$ perovskite solar cells. Nat. Commun. 2016, $7,10334$.

(32) Liu, S.; Zheng, F.; Grinberg, I.; Rappe, A. M. Photoferroelectric and Photopiezoelectric Properties of Organometal Halide Perovskites. J. Phys. Chem. Lett. 2016, 7, 1460-1465.

(33) Xing, J.; Wang, Q.; Dong, Q.; Yuan, Y.; Fang, Y.; Huang, J. Ultrafast ion migration in hybrid perovskite polycrystalline thin films under light and suppression in single crystals. Phys. Chem. Chem. Phys. 2016, 18, 30484-30490.

(34) Yuan, M.; Quan, L. N.; Comin, R.; Walters, G.; Sabatini, R.; Voznyy, O.; Hoogland, S.; Zhao, Y.; Beauregard, E. M.; Kanjanaboos, P.; et al. Perovskite energy funnels for efficient light-emitting diodes. Nat. Nanotechnol. 2016, 11, 872-877.

(35) Tian, W.; Leng, J.; Zhao, C.; Jin, S. Long-Distance Charge Carrier Funneling in Perovskite Nanowires Enabled by Built-in Halide Gradient. J. Am. Chem. Soc. 2017, 139, 579-582.

(36) Jesper Jacobsson, T.; Correa-Baena, J.-P.; Pazoki, M.; Saliba, M.; Schenk, K.; Grätzel, M.; Hagfeldt, A. Exploration of the compositional space for mixed lead halogen perovskites for high efficiency solar cells. Energy Environ. Sci. 2016, 9, 1706-1724.

(37) Jeon, N. J.; Noh, J. H.; Yang, W. S.; Kim, Y. C.; Ryu, S.; Seo, J.; Seok, S. Il. Compositional engineering of perovskite materials for highperformance solar cells. Nature 2015, 517, 476-480.

(38) Neutzner, S.; Srimath Kandada, A. R.; Lanzani, G.; Petrozza, A. A dual-phase architecture for efficient amplified spontaneous emission in lead iodide perovskites. J. Mater. Chem. C 2016, 4, 4630-4633. 